\title{
Die digitale Konstellation. Eine Positionsbestimmung
}

\author{
Sebastian Berg • Niklas Rakowski • Thorsten Thiel
}

Online publiziert: 6. März 2020

(C) Der/die Autor(en) 2020

Zusammenfassung Die Entwicklung der digitalen Gesellschaft ist in den Sozialwissenschaften $\mathrm{zu}$ einem zentralen Forschungsgegenstand aufgestiegen; in der Politikwissenschaft gilt allerdings, dass die Auseinandersetzung auf eher wenige Schwerpunkte - insbesondere Digitalisierung als vernetzte Kommunikation - beschränkt geblieben ist. Der Beitrag macht einen Vorschlag für eine andere Form der politikwissenschaftlichen Auseinandersetzung indem er - nach einer kritischen Rekonstruktion der Forschungsentwicklung in der Politikwissenschaft - breit rezipierte Diagnosen der digitalen Gesellschaft in Nachbardisziplinen wie der Soziologie und Rechtswissenschaft (Baecker, Nassehi, Reckwitz, Stalder und Vesting) rekonstruiert. Er arbeitet übergreifende Erkenntnisse im Digitalisierungsverständnis dieser Ansätze heraus, kritisiert jedoch deren Konzeptualisierung von Politik und Demokratie. Auf dieser Grundlage wird sodann ein Vorschlag unterbreitet, eine in der Politikwissenschaft anschlussfähigere Perspektive mittels des Konzepts der „digitalen Konstellation“ zu entwickeln. Dieses untersucht das Wechselspiel technischer und gesellschaftlicher Entwicklungen in Bezug auf den Zusammenhang zum Formwandel von Demokratie. Abschließend wird dies in einer exemplarischen Analyse von Veränderungen des Repräsentationskonzepts im digitalen Kontext veranschaulicht.

S. Berg · N. Rakowski $\cdot$ T. Thiel $(\bowtie)$

Forschungsgruppe Demokratie und Digitalisierung, Weizenbaum-Institut für die vernetzte

Gesellschaft, Wissenschaftszentrum Berlin für Sozialforschung, Hardenbergstraße 32, 10623 Berlin,

Deutschland

E-Mail: thorsten.thiel@wzb.eu 


\title{
The digital constellation. Rethinking political perspectives
}

\begin{abstract}
The emergence of the digital society has become one of the most pressing research topics in social science. So far political science has been at the margins of the debate being restricted by a rather narrow focus on networked communications. The paper attempts to change this by presenting a more encompassing way to thematize digitalization from within political science. After briefly having criticized the research development in political science the paper reconstructs at length some of the most popular conceptualizations in neighboring disciplines. While we highlight the commonalities and strengths of those approaches in theorizing digitalization, we criticize their rather derivative understanding of democratic practices and the political as such. We go on to propose a modified understanding-labeled the "digital constellation"- that looks at the changing shape of democracy by developing a much more nuanced understanding of the interplay of societies and technology. Finally, the argument is illustrated by a short exemplary analysis of the changes occurring in political representation in the context of digitalization.
\end{abstract}

\section{Einleitung}

Digitale Technik als eine bedeutsame transformative Kraft zu begreifen und den Wandel in Alltag, Politik, Wirtschaft und nahezu allen weiteren Bereichen gesellschaftlichen Lebens rhetorisch auf sie zu beziehen, ist eine seit vielen Jahren gängige, längst zum Klischee gewordene Praxis. Während lange Jahre vor allem die transformative Kraft des Internets Gegenstand der Betrachtung war, gehen gerade jüngere Ansätze über die unmittelbare Informations- und Kommunikationstechnik hinaus und schauen stattdessen umfassend auf die Rekonstruktion gesellschaftlicher Praktiken - und damit die Verfasstheit unserer Gesellschaft als digitale Gesellschaft. Auffallend ist: Trotz vielfältiger Bezüge findet die ausufernde Debatte um Digitalisierung und Demokratie dabei bisher ganz überwiegend jenseits der Politikwissenschaft statt.

Um dies zu ändern und Politikwissenschaft - insbesondere Politische Theorie anschlussfähiger an die Diskussion um Digitalisierung und Gesellschaft zu machen, geht der Beitrag auf zwei Weisen vor: Wir unternehmen eine doppelte Aufarbeitung des Forschungsstandes, zunächst kurz in Bezug auf die politikwissenschaftliche Auseinandersetzung, sodann ausführlicher im Hinblick auf zentrale Beiträge aus benachbarten Disziplinen. Für letztere rekonstruieren wir, wie diese Digitalisierung verstehen, welche Theorien der digitalen Gesellschaft sie entwickeln und wie sie sich in diesen auf das Politische bzw. die Demokratie beziehen. Dies aufnehmend, aber dann politikwissenschaftlich wendend, unterbreiten wir im dritten Teil des Textes den Vorschlag, im Anschluss an Jürgen Habermas den Konstellationsbegriff zum Ausgangspunkt einer politikwissenschaftlichen Perspektive auf die digitale Konstellation zu machen. Anders als in den Großtheorien der digitalen Gesellschaft wollen wir in dieser Konzeption Demokratie und das Politische weniger subsumierend und reaktiv erfassen, sondern genauer herausstellen, wie das Wechselspiel von techni- 
schen und gesellschaftlichen Entwicklungen die Möglichkeiten, Voraussetzungen und Formen von Demokratie verändert.

\section{Digitalisierung als Forschungsgegenstand in der Politikwissenschaft}

Digitalisierung als trending topic ist selbstverständlich nicht spurlos an politikwissenschaftlichen und demokratietheoretischen Debatten vorbeigegangen (einen Überblick gibt: Kersting 2019). Wir wollen im Folgenden aber argumentieren, dass die bisherige Diskussion des Themas in mehrerlei Hinsicht begrenzt ist und deshalb letztlich zu kurz greift.

Ausgangspunkt des politikwissenschaftlichen Interesses an Digitalisierung ist die Vernetzungstechnik des Internets, deren disruptive gesellschaftliche Wirkung in der zweiten Hälfte der 90er Jahre in Politik wie Öffentlichkeit offenbar und dann auch sehr unmittelbar zum Gegenstand wissenschaftlicher Reflexion wurde. Für die demokratietheoretisch orientierte - Politikwissenschaft steht diese technischen Veränderung im direkten Zusammenhang mit den zu jener Zeit vehement geführten Diskussionen um neue Weisen des Regierens, um Globalität und die Hoffnung auf eine Demokratisierung der Demokratie (Bohman 2004). Neue Governance-Modelle, die als stark inklusiv und aktiv-partizipativ gedacht werden, prägen die Diskussion; auch wenn bereits ab der initialen Phase technikkritische Argumentationen einen festen Platz im demokratiebezogenen Diskurs haben, zumal in Deutschland (Buchstein 1996; Schmalz-Bruns 2001). Diskutiert wird, ob die sich durch digitale Techniken verändernden Möglichkeiten gesellschaftlicher Partizipation revolutionär sind oder nur bestehenden Eliten nützen (Barber 1998), ob das Netz eine öffentliche Sphäre ist (Dean 2003) oder wie sich die Potentiale vernetzter Kommunikation verwirklichen lassen (Dahlgren 2009). Der überwiegende Teil der Literatur arbeitet sich dabei direkt am Beteiligungsversprechen bzw. dessen (Nicht-) Einlösung ab (Hindman 2008). ${ }^{1}$

Die wichtigste Iteration dieser Perspektive setzt in der Folge des Aufstiegs sozialer Medien ab ca. 2005 ein. Diese zunächst als Web 2.0 apostrophierte Veränderung bewirkt, dass die Vernetzung und Kommunikation zwischen Bürger*innen stärker in den Vordergrund tritt und sich der vorherige Schwerpunkt auf Information (Transparenz und Erreichbarkeit) variiert. Zu der Annahme einer im positiven Fall responsiver werdenden institutionellen Politik und direkter involvierten Bürger*innen, treten nun vor allem Untersuchungen zu den neuen Möglichkeiten der Selbstorganisation von Kollektiven (Bennett und Segerberg 2014; Earl und Kimport 2011). Die Idee eines digitalen Strukturwandels von Öffentlichkeit wird immer prominenter und erreicht einen Kulminationspunkt in der Beschäftigung mit den Platzrevolutionen vom Arabischen Frühling bis hin zur Occupy-Bewegung (Howard 2010; Tufekci 2017) und der Auseinandersetzung mit Phänomenen wie der Piratenpartei (Bieber und

\footnotetext{
1 Die Debatten um Demokratie und das Internet sind selbstverständlich oft in Form von Artikeln (z. B. Margetts 2013) oder Sammelbänden (etwa. Kneuer 2013; Kneuer und Salzborn 2016; Jacob und Thiel 2017) zusammengefasst worden. Ein schon älterer, aber immer noch guter Artikel, der expliziter auf Demokratietheorie und Politische Theorie eingeht, ist: Dahlberg 2011.
} 
Leggewie 2012). Demokratiegefährdende, insbesondere die gesellschaftliche Fragmentierung und politische Polarisierung vorantreibende Mechanismen werden dabei von Anfang an gesehen (Sunstein 2008; Habermas 2008; Keane 2013) und sind dann insbesondere in der Forschung zu Filterblasen, Fake News und Hate Speech zentral geworden (Margetts 2019).

Jenseits des Schwerpunkts auf Partizipation/Repräsentation und Kommunikation/Öffentlichkeit haben andere Bereiche demokratietheoretisch relevanter digitaler Veränderung relativ wenig Aufmerksamkeit erhalten, wobei Ausnahmen die Regel bestätigen: etwa der Diskurs um Privatheit (Seubert 2016; Thiel 2017), die Auseinandersetzung mit der Veränderung staatlicher Souveränität (Pohle und Thiel 2019; Ritzi und Zierold 2019), die Erforschung digitalen zivilen Ungehorsams (Celikates 2015; Kleger und Makswitat 2014) oder Arbeiten zu Überwachung durch öffentliche oder privatwirtschaftliche Akteure (Stahl 2016). Hinzu kommen anlassbezogene, meist aber eher kurzfristige Beschäftigungen etwa in Bezug auf die Rolle von Geheimdiensten in Folge der Snowden-Enthüllungen (Steiger et al. 2017) sowie bereichsspezifische Arbeiten zu Netzpolitik (Reiberg 2018; Busch et al. 2019), E-Government (Schünemann und Kneuer 2019) oder der Entwicklung demokratischer Parteien (Jäckel und Jun 2015). ${ }^{2}$ Für all diese Arbeiten gilt aber, wie schon für die Auseinandersetzung um Partizipationswandel, dass das Verständnis von Digitalisierung auf die Vernetzungslogik des Internets fixiert ist.

Festzuhalten ist außerdem, dass in jüngerer Zeit sich in der Politikwissenschaft immer stärker ein empirischer Zugriff auf Digitalisierung als Forschungsgegenstand durchgesetzt hat. In diesem wird der Schwerpunkt auf Partizipation und Öffentlichkeit gelegt und Vernetzung stets als das zentrale Element verstanden, welches dynamisch und verstärkend wirkt und somit demokratiefördernd oder demorkatieunterwandernd sein kann - fraglich ist allein zu welchen Teilen und in welchen Kontexten. Anders als in der frühen Literatur, die oft abstrakt das Internet als Wirkmacht benennt, wird in diesem Forschungszweig stärker kontextualisiert und die Aussagen werden auf einzelne Techniken bzw. Plattformen heruntergebrochen. Der empirische Ansatz, welcher zudem von neuen Datenquellen und digitalen Methoden profitiert, reklamiert für sich einen Professionalisierungsgewinn, der die Debatte aus der feuilletonistischen Diskussion zieht und verwissenschaftlicht (Jungherr und Theocharis 2017). Der Erfolg des Ansatzes bringt aber auch die Gefahr mit sich, den Blick der Politikwissenschaft für Digitalisierung als gesellschaftliches Phänomen noch weiter zu verengen. Dies steht in einem starken Kontrast zum öffentlichen Diskurs, in dem der Fokus auf das Internet als Kommunikationstechnik zugunsten eines stärker holistischen Digitalisierungsdiskurses abgelöst wurde und in dem verstärkt die Regulierung des Digitalen diskutiert wird, nicht nur dessen Auswirkungen.

\footnotetext{
${ }^{2}$ Eine hier nicht zu behandelnde, wenn auch durchaus umfangreiche politikwissenschaftliche Auseinandersetzung mit Digitalisierung findet zudem noch im Teilbereich der Internationalen Beziehungen statt. Obwohl hier selbstverständlich auch gelegentlich Fragen der Normsetzung oder der Demokratiestabilität eine Rolle spielen, ist diese Literatur für die in diesem Text interessierende Fragestellung irrelevant, da sie nicht die innergesellschaftlichen Re-Konfigurationen zum Thema macht und Digitalisierung zumeist nicht in ihrer Wirkweise zu verstehen sucht, sondern als Domäne (Cyberspace) oder besondere Variable im Machtspiel der Staaten interpretiert.
} 
Zusammenfassend lässt sich festhalten, dass die politikwissenschaftliche Auseinandersetzung mit Digitalisierung diese überwiegend als Kommunikations- und Informationstechnik versteht und damit auf ihre Vernetzungswirkung reduziert. Sie fokussiert sodann in der Hauptsache die Schnittstelle von deliberativer Öffentlichkeit und repräsentativer Demokratie. Im Vergleich zur allgemeinen Debatte um Digitalisierung ist dies ein relativ enger Fokus, der selbst sich abzeichnende Megatrends wie maschinelles Lernen und künstliche Intelligenz nur unzureichend aufnimmt (s. aber: Ulbricht et al. 2018) und die vielfältigen Wirkungen von Digitalität in Gesellschaft unterschätzt.

\section{Entwürfe der digitalen Gesellschaft}

Schaut man über die Ränder der akademischen Disziplin Politikwissenschaft auf die Debatten in den weiteren Sozialwissenschaften, so fällt unmittelbar auf, dass dort sehr viel breiter die Transformation hin zur digitalen Gesellschaft in den Blick genommen wird. Digitalisierung wird als eine gesamtgesellschaftliche Veränderung thematisiert und der Fokus auf Kommunikationsmöglichkeiten zunehmend durch ein Nachdenken über andere digital konnotierte Veränderungen - wie etwa Automatisierung - ergänzt. Stärker als in der Politikwissenschaft wird ein Zusammenhang von technischem Wandel und einer allgemeinen Umstrukturierung gesellschaftlicher Rationalitäten thematisiert, welcher dann auch als unmittelbar bedeutsam für Politik interpretiert wird. Diese Debatten sollen im Folgenden exemplarisch rekonstruiert werden, um zu prüfen, inwiefern die dort verhandelten Entwicklungen für die politikwissenschaftliche Aufarbeitung anschlussfähig sind und welches Politik- und Demokratieverständnisse sich in den politikwissenschaftlichen Diskurs übertragen ließe.

Für die hier interessierende Frage ist es dabei nicht notwendig, einen Gesamtüberblick über die sehr umfangreiche und dynamische Forschungsentwicklung zu geben. Vielmehr haben wir uns bewusst dafür entschieden, zentrale Texte gerade der deutschsprachigen Diskussion um die digitale Gesellschaft auszuwählen. Als Kriterien haben wir angelegt, dass es sich um theoretisch orientierte, gesamtgesellschaftliche Fragen aufwerfende Monographien handeln soll, die in den letzten fünf Jahren erschienen, auf größere Leser*innenschaften hin angelegt sind und in der öffentlichen Diskussion breit rezipiert wurden. Hierbei sticht hervor, dass gerade die Soziologie das Thema stark für sich reklamiert und entwickelt hat - zum einen im Bereich der Allgemeinen Soziologie, zum anderen im Feld der Kultursoziologie bzw. den Kulturwissenschaften. Aus dieser Beobachtung heraus haben wir mit dem Werk von Thomas Vesting einen passenden rechtswissenschaftlichen Beitrag in die Betrachtung aufgenommen. ${ }^{3}$ Die ausgewählten Texte sind Fixpunkte in der deutschsprachigen Diskussion um die digitale Gesellschaft, sie fassen jeweils selbst

\footnotetext{
3 Selbstverständlich hätte es die Möglichkeit gegeben, eine breitere Auswahl an Autor*innen zu treffen und diese auch interdisziplinärer zu gestalten. So ließe sich etwas aus den Kommunikations- und Medienwissenschaft, die Monographie von Nick Couldry und Andreas Hepp „The Mediated Construction of Reality“ (2016) anführen oder aus der Philosophie wäre Luciano Floridis auch ins Deutsche übersetztes Buch „Die 4. Revolution“ (2015) einschlägig gewesen. Dass wir diese und weitere Werke nicht explizit mit
} 
Forschungsstände zusammen und transportieren den Anspruch, auch Aufschluss über die Entwicklung von Politik und Demokratie zu geben. ${ }^{4}$

Im Folgenden wollen wir zunächst rekonstruieren, wie diese Texte Digitalisierung/Digitalität und die Entwicklung zur digitalen Gesellschaft konzeptualisieren, bevor wir auf deren jeweiliges Verständnis von Politik und dem Politischen eingehen.

\subsection{Digitalisierung, Digitalität und digitale Gesellschaft}

Die Hinwendung zur Thematik der digitalen Gesellschaft wird am prononciertesten in der Soziologie vorgenommen. Hier haben in den letzten Jahren eine große Zahl an Arbeiten versucht, eine umfassende Theorie der digitalen Gesellschaft vorzulegen. Zwei Arten des Zugriffs sind zentral: Ein Zugriff, der in der Allgemeinen Soziologie $\mathrm{zu}$ verorten ist und in dem ein starker systemtheoretischer Einschlag kennzeichnend ist, und ein stärker an Kultur und Praktiken orientiertes Argument, welches die Gesellschaftsentwicklung in der Folge veränderter Handlungsmöglichkeiten erklärt.

Für den systemtheoretischen Zugriff sind die Werke von Dirk Baecker „4.0 oder Die Lücke die der Rechner lässt“ (2018) und Armin Nassehi „Muster: Eine Theorie der digitalen Gesellschaft“ (2019) einschlägig. Gemeinsam ist diesen ein Verständnis von Digitalisierung, das die Form des Digitalen unmittelbar mit dem Prozess der gesellschaftlichen Selbsterfassung kombiniert. Ein Begriff von Digitalisierung, der also bereits abstrakt ist und sich auf der definitorischen Ebene von technischen Aspekten sehr weitreichend löst. Bei Baecker wird Digitalisierung etwa gefasst als „Erarbeitung und Erprobung abzählbarer und berechenbarer Daten im Medium analoger Widersprüchlichkeit für die Zwecke der Kommunikation von und mit Maschinen“ (Baecker 2018, S. 59) und auch Armin Nassehi sieht das Kernprinzip des Digitalen in der radikalen Vereinfachung der binären Codierung, welche dann aufgrund ihres unspezifischen Charakters eine universale und deshalb unendlich vielschichtige Anwendung erlaubt (Nassehi 2019, S. 147). In der Durchsetzung der Form des Digitalen im gesellschaftlichen Prozess ergibt sich für beide Autoren dann der eigentlich interessante Effekt der Digitalisierung: Diese verdopple Gesellschaft, indem sie die analoge Wirklichkeit zunächst in binäre Unterscheidungen codiert und dann die sich daraus ergebenden Daten an diesen Unterscheidungen selbst erprobt (Nassehi 2019, S. $107 \mathrm{ff}$.).

Baecker ruft ob dieser rekursiven Form, in der Maschinen, statistische Prozesse und andere Formen der Automatisierung aktiv in die gesellschaftliche Sinnproduk-

eigenen Wiedergaben in die Betrachtung aufgenommen haben, ist nicht als kategorisches oder qualitatives Urteil zu verstehen. Vielmehr haben wir uns für eine stärker homogene Auswahl der bearbeiteten Literatur entschieden, die sich besser für eine pointierte Diskussion eignet.

4 Es sei explizit darauf hingewiesen, dass unsere Auswahl ausschließlich auf männliche Autoren verengt. In der internationalen Debatte sind weibliche Stimmen durchaus prägend (etwa José van Dijck oder Antoinette Rouvroy), diese haben jedoch einen weniger allgemeinen Erklärungsanspruch. Eine potentielle Autorin in der Logik unserer Ausarbeitung wäre einzig Shoshanna Zuboff gewesen, deren „,Das Zeitalter des Überwachungskapitalismus“ (2018) ein zentrales Werk der Diskussion der vergangenen Jahre ist. Hier haben wir uns aufgrund seines ökonomiezentrierten Zuschnitts dagegen entschieden, es in die detaillierte Wiedergabe aufzunehmen. Das von uns später vorgetragene Argument einer unterkomplexen Theoretisierung des demokratischen Prozesses, wäre jedoch auch in der Auseinandersetzung mit Zuboff $\mathrm{zu}$ formulieren gewesen. 
tion einbezogen werden, eine vierte Medienepoche aus. In dieser gehe es eben nicht mehr nur um die Verbreitung von Information, was Gegenstand der dritten Epoche und des Buchdrucks als deren technischer Revolution gewesen sei. Der revolutionäre Charakter der vierten Epoche bestehe in der quasi unbegrenzten Rekombinierbarkeit von Daten. ${ }^{5}$ Armin Nassehis Ansatz kehrt diese Perspektive in einem gewissen Sinne um, da er zunächst nicht nach der Zukunft der digitalen Gesellschaft fragt, sondern nach deren Grund: Für welches gesellschaftliche Problem ist Digitalisierung eigentlich die Lösung (Nassehi 2019, S. 12)? Nassehi zufolge ist Digitalisierung nämlich nur deshalb als persistente und durchgesetzte Technik zu denken, weil ihr Funktionieren gesellschaftlichen Sinn erfüllt. Konkret, weil das Bezugsproblem der Digitalisierung die Komplexität und vor allem die Regelmäßigkeit der Gesellschaft selbst ist. Komplexität resultiert Nassehi zufolge nicht ursprünglich aus digitalen Techniken, sie entsteht bereits vorgängig im Zuge eines sich wandelnden gesellschaftlichen Selbstverständnisses und findet daher etwa auch im vor-digitalen Aufkommen der Soziologie schon einen Ausdruck.

Aber egal, ob digitale Techniken revolutionärer Treiber oder reaktive Funktion im Angesicht gesellschaftlicher Komplexität sind, Nassehi und Baecker treffen sich wieder, wenn sie als Folge der Durchsetzung digitaler Technik einen Kontrollüberschuss diagnostizieren (Baecker 2018 14ff.; Nassehi 2019, S. 43). Weil Digitalisierung als funktional notwendige Antwort auf Komplexität digitale Welterfassung selbst immer weiter perpetuiert und in den Datenfüllen immer deutlicher Muster zutage treten, derer die Gesellschaft selbst ohne die Instrumente digitaler Erkenntnis nicht gewahr ist, wird diese anders und vehementer zur Beschäftigung mit sich selbst gezwungen. Es entsteht ein Veränderungsdruck, der alle Teile der Gesellschaft erfasst und welcher die Gewissheiten einer zielorientierten rationalistischen Moderne unterminiert. Nach Baecker generiert dies einen katastrophischen Zustand, eine tiefe Form der Ungewissheit, da die kommende Gesellschaft erst wieder adäquate Formen und Strukturen finden muss. So werde funktionale Differenzierung als Kennzeichen der Moderne abgelöst, Inklusion und Exklusion fortan durch die flexiblen Ein- und Ausschlüsse des Netzwerks adressiert (Baecker 2018, S. $26 \mathrm{ff}$.). Nassehi hingegen besteht zwar auf dem Fortwirken der Differenzierung, arbeitet jedoch umfassend heraus, wie die vormalige Illusion, dass die differenzierte Gesellschaft letztlich doch über ein Arrangement politischer Institutionen gesteuert werden könne, in der radikalen Vielfalt ihrer offenbar werdenden praktischen Möglichkeiten und Variationen vergehe (Nassehi 2019, S. 185).

Die zweite große Diskussionslinie in der Soziologie hat zwar einen kaum weniger umfassenden Erklärungsanspruch, macht ihr Argument jedoch stärker auf einer handlungstheoretischen anstatt einer komplexitätstheoretischen Grundlage. Für einen solchen Ansatz stehen die Bücher „Die Gesellschaft der Singularitäten“ von Andreas Reckwitz (2017) und die „Kultur der Digitalität“ von Felix Stalder (2016).

\footnotetext{
5 Armin Nassehi periodisiert dies anders, indem er nicht Medienkonstellationen, sondern die gesellschaftliche Selbstreflexion als Bezugspunkt wählt: Für ihn stellt Digitalisierung dann die dritte Entdeckung der Gesellschaft dar. Die erste Entdeckung war die Geburt der Nationalstaaten, die zweite die Liberalisierung und Pluralisierung seit Mitte des 20. Jahrhunderts (Nassehi 2019, S. 45 ff.).
} 
Das Argument von Andreas Reckwitz bildet hierbei zunächst einmal eine Brücke zu den vorangestellten Ansätzen, ist doch dessen zentrales Erkenntnisinteresse ebenfalls die Erfassung und Begründung eines umfassenden Strukturwandels der Spätmoderne. In diesem verliert, so Reckwitz zentrale These, die ,soziale Logik des Allgemeinen ihre Vorherrschaft [...] an die soziale Logik des Besonderen " (Reckwitz 2017, S. 11). Digitalisierung ist in diesem Prozess eine von drei Triebfedern: Sie wird erst in der Koinzidenz mit der ,,sozio-kulturelle[n] Authentizitätsrevolution“ und der ,Transformation der Ökonomie hin zu einer postindustriellen Ökonomie der Singularitäten“ (Reckwitz 2017, S. 103) zu jener Kraft, die die Gesellschaft als Ganze revolutioniert. Die spezifische Bedeutung von Digitalisierung in den Ausführungen von Reckwitz ist dabei, dass mittels ihrer die generalisierenden Techniken der industriellen Moderne in der „Kulturmaschine des Internets“ aufgehen und darüber ihre egalisierende und standardisierende soziale Prägekraft verlieren. Digitalisierung fungiert als infrastrukturelle Ermöglichungsbedingung kultureller Singularisierungspraktiken, die weniger auf gesamtgesellschaftliche Komplexitätsbewältigung als auf individuelle Sinnstiftung abzielen (Reckwitz 2017, S. 103, $229 \mathrm{ff}$., vgl. $308 \mathrm{ff}$.). Der Wandel gesellschaftlicher Strukturen begründet sich damit nicht wie bei Baecker über die strukturelle Eigenlogik digitaler Technik, sondern über das handlungslogische Zusammenkommen gesellschaftlicher Praktiken - „,doing generality“, ,doing singularity“ - mit den Affordanzen digitaler Technik (sowie des ökonomischen und kulturellen Kontexts) (Reckwitz 2017, S. 225).

Wie dies funktioniert, wird noch deutlicher, wenn man sich dem Ansatz von Stalder zuwendet, der unmittelbar ein handlungstheoretisches Verständnis des digitalen Wandels expliziert - und dies nicht nur für ein größeres Argument im Kontext des Strukturwandels benötigt. Stalder zufolge muss Digitalität als neue ,kulturelle Konstellation“ begriffen werden (Stalder 2016, S. 11f.). Sie bezeichnet ,jenes Set von Relationen, das heute auf der Basis der Infrastruktur digitaler Netzwerke in Produktion, Nutzung und Transformation materieller und immaterieller Güter sowie in der Konstitution und Koordination persönlichen und kollektiven Handelns realisiert wird" (Stalder 2016, S. 18). Wie schon bei Baecker und Nassehi geht Digitalisierung daher über das technisch-mediale Moment hinaus, nun aber, indem sie als ein „relationales Muster“ der intersubjektiven und interobjektiven Kommunikation gewertet wird, in der ,historisch neue Möglichkeiten der Konstitution und der Verknüpfung der unterschiedlichsten menschlichen und nichtmenschlichen Akteure“ entstehen (Stalder 2016, S. 18). Daraus folgt zum einen, dass die „Präsenz der Digitalität [auch] jenseits der digitalen Medien“ besteht (Stalder 2016, S. 19), zum anderen, dass Technik nicht nur die Bedingungen kultureller Praktiken konfiguriert, sondern vielmehr selbst aus widersprüchlichen und kontingenten Verfahren bzw. der Erfahrung der sozialen Nutzung kreiert und stabilisiert wird (Stalder 2016, S. 17). Für Stalder sind es dabei insbesondere drei spezifische kulturelle Formen, die die Kultur der Digitalität auszeichnen: Referentialität, Gemeinschaftlichkeit und Algorithmizität (Stalder 2016, S. 95 ff.).

Bevor wir übergehen zu dem Politikbegriff, der aus diesen soziologischen Zugriffen folgt, sei noch ein Blick in die Rechtswissenschaft, als weitere, für die Politikwissenschaft besonders wichtige Nachbardisziplin geworfen. Auch hier ist in jüngerer Zeit ein neues Problembewusstsein für die digitale Gesellschaft und ihre Herausfor- 
derung für das rechtlich-politische Arrangement der Gegenwart aufgekommen. Der Zugriff in der Rechtswissenschaft unterscheidet sich dabei schon deshalb von jenem der Soziologie aber auch der Politikwissenschaft, da in der Rechtswissenschaft deutungsstarke prognostische Ansätze nicht im gängigen Diskurs des Faches anzutreffen sind. Hier stellen allein die Fragen nach der Möglichkeit von Regulierung technischer Innovationen (Eifert und Hoffmann-Riem 2009) oder der Ausgestaltung spezifischer Rechtsinstitute unter dem Vorzeichen der Digitalisierung (Fries 2016) den gängigen Zugang dar. Trotzdem finden sich auch hier Beiträge, welche eine gesamtgesellschaftliche und auch demokratietheoretische Reflexion vornehmen und daher im Kontext dieses Beitrags von Interesse sind. Das beste Beispiel hierfür ist der Abschlussband der vierbändigen Buchreihe „Medien des Rechts“ von Thomas Vesting (2015) (vgl. aber auch: Kersten 2017; Ingold 2017).

Nach Vesting erschöpft sich die Wirkung von Medien wie Sprache, Schrift, Buchdruck oder eben Computernetzwerken nicht allein in ihrer Funktion als Mittler, sondern in deren formgebenden Wirkung, in der sich ,,bedeutungstragende Strukturen und Ordnungen eintragen“ (Vesting 2015, S. 50). Medien reproduzieren somit nicht einfach Recht, sondern schaffen einen kulturellen Rahmen, aus dem das Recht erst seine Normativität generieren kann.

Anders als die soziologischen Autoren ist Vesting dabei weniger abstrakt, was Wirkung und Form von Digitalität angeht. Für ihn zentral ist die Bedeutung des Computers als materielles Artefakt, welches in der Lage ist, Medien miteinander zu verflechten (Vesting 2015, S. 53). Durch den Zusammenschluss von Computern zu Netzwerken entstehe dann jene dynamische Konstellation, deren Aufkommen einen kulturellen Wandel ,,von einer hierarchisch-zentralistischen zu einer heterarchisch-azentrischen Kultur" bewirke (Vesting 2015, S. 56) und innerhalb derer sich neue, paradigmatische Formen der Kommunikation herausbilden: die flüchtige, affektgeladene Aufladung digitaler Kommunikation etwa, die Mikrodiversität sozialer Medien oder die erschwerte Grenzziehung zwischen politischen Ereignissen und Medienereignissen. Auch bei Vesting mündet eine „Kultur der Netzwerke“ daher in einem revidierten Verständnis von sozialer Epistemologie und Subjektivität. Beides könne in der Netzwerkgesellschaft nur noch relational bestimmt werden (Vesting 2015, S. 61, 72 ff.). Dies habe auch Folgen für das Recht und rechtliche Normen, es entsteht ein „Recht der Netzwerkkultur“. Dieses ersetzt die vorherigen Rechtsstrukturen nicht, fügt ihnen jedoch weitere Schichten und Ebenen hinzu. Kennzeichnend für das Recht der Netzwerkkultur ist, dass es auf die abnehmende Bindungswirkung von Gruppenidentitäten und die Individualisierungstendenz der Subjekte Antworten finden muss (Vesting 2015, S. $153 \mathrm{ff}$.).

\subsection{Politik: Epiphänomen und Medienprodukt}

Die Institutionen und Prozesse der Politik haben in den fünf nunmehr kurz vorgestellten Ansätzen jeweils nur eine nachgelagerte Bedeutung. Dies liegt zunächst einmal an den disziplinären Interessen der Autoren, bspw. in der soziologischen Umschreibung von Politik als eines spezifischen gesellschaftlichen Teilbereichs. Darüber hinaus ist es aber die Konzeptualisierung von Digitalisierung selbst, die nach unserer Auffassung zu einem zu weitgehenden Aberkennen der Bedeutung kollekti- 
ver politischer Handlungsformen führt - und es ist dieser Aspekt der Analysen, den wir nun noch einmal gesondert herausarbeiten und kritisieren wollen.

Bei Thomas Vesting geschieht die Rückstufung recht unmittelbar durch das starke Setzen der Begrifflichkeit des Netzwerks. Das Netzwerk wird bei Vesting eben nicht nur als kommunikative Form gesehen, sondern zum Strukturmerkmal jedweder politischen Institutionalisierung erklärt. So fordert er etwa, die repräsentativen Strukturen der Demokratie auf die neue Netzwerkkultur hin auszurichten, z. B. durch die Etablierung digitaler Partizipationsformen oder durch eine Abkehr von der parteiförmigen Organisation repräsentativer Politik (Vesting 2015, S. 158). Durch die „Stärkung von Institutionen der Selbstorganisation netzwerkartiger Handlungszusammenhänge“ (Vesting 2015, S. 162) adaptiert Vesting eine technische Metaphorik der Dezentralisierung. Diese medientheoretische Deutung überzeichnet dabei Politik als Abbild des medialen Wandels und verpasst es, den kontingenten und konfliktiven Charakter technischer Adaptionsprozesse anzuerkennen.

In den soziologischen Diagnosen der Politik im Kontext der digitalen Gesellschaft ist es nicht ein Imperativ der Nachbildung der digitalen Struktur, sondern ein ganz allgemeiner, wenn auch in allen Ansätzen unterschiedlich zum Ausdruck gebrachter Zweifel an der Leistungsfähigkeit politischer Institutionen, der die Kritik prägt.

Für die beiden Ansätze in systemtheoretischer Tradition - Nassehi und Baecker ist dies zunächst einmal nur die Bewahrheitung einer ohnehin schon alten Erkenntnis der Systemtheorie: der Relativität politischer Steuerung. ${ }^{6}$ Nassehi illustriert dies in zweifacher Hinsicht: Einmal, wenn er aufzeigt, wie die radikale Optionssteigerung in den Teilsystemen und die gesamtgesellschaftliche Komplexitätssteigerung allgemein Politik störungsanfällig macht (Nassehi 2019, S. 183), zum anderen wenn er bereichsspezifische Entwicklungen der Digitalisierung, etwa die Plattformökonomie oder Big Data, so interpretiert, dass diese den demokratischen Modus der kollektiven politischen Steuerung der Gesellschaft als Ganzen herausfordern. Funktionale Differenzierung ernst zu nehmen bedeutet im Kontext der digitalen Gesellschaft, sie nicht mehr hinter einem generalisierenden Institutionenarrangement unsichtbar machen zu können (Nassehi 2019, S. 185). Eine durch Digitalisierung gekennzeichnete Gesellschaft sei angesichts der entfalteten Komplexität bei gleichzeitigem Gewahr werden der Musterhaftigkeit kollektiven Handelns für alle erkennbar dezentriert. Sie könne daher nur noch durch verteilte gesellschaftliche Prozesse beeinflusst, nicht mehr in irgendeiner Weise kollektiv-zentral gesteuert werden. Eine angemessene Politik ist daher nur als stark responsive, zurückgenommene denkbar, die direkt mit der digitalen Infrastruktur als Assemblage agiert.

Dirk Baecker geht zwar in ähnlicher Weise davon aus, dass Politik angesichts von Komplexität überfordert sei und nicht anders funktionieren könne als durch eine stete „Reformatierung ihrer Möglichkeiten im Medium des Zerfalls und des Wiederaufbaus jeder Art von Wirklichkeit“" (Baecker 2018, S. 97); anders als Nassehi versucht er sich aber sehr wohl an einer Prognose, wie Politik sich konkret verändern werde: Ausgangspunkt ist seine Bestimmung, dass Politik immer auch das ist, was ,das

\footnotetext{
6 Diese Einsicht findet ihren Ausgang wiederum in den kybernetischen Deutungsansätzen der Gesellschaft, welche das ältere souveränitätszentrierte Denken des Politischen für die Moderne als inadäquat beschrieben und ideenpolitische Vorläufer des Netzwerksdenkens bilden (August 2018; vgl. Deutsch 1969).
} 
Publikum von der Politik erwartet“ (Baecker 2018, S. 97). Dieser Schwenk zu einem kommunikativen Politikbegriff wird sodann stark aufgeladen, wenn Baecker als zentralen Gegenstand politischer Kommunikation die Entscheidung zwischen Krieg und Frieden bzw. Einschluss und Ausschluss setzt (Baecker 2018, S. 101). Politik in Baeckers Verständnis ist der Kampf um Macht; die Möglichkeit, Drohungen zu realisieren, um andere zu Folgsamkeit zu zwingen. Die Bedingungen der Machtausübung verschärfen sich dabei in der digitalen Gesellschaft, da der Kontext der Politik affektgeladener sei und die Möglichkeiten der Manipulation zunehmen (Baecker 2018, S. 103). Dieser, im Kontext des Buches überraschende, weil doch schon ältere Politikbegriff wird zum Schluss des betreffenden Kapitels dann in einer kurzen Reflexion über die möglichen institutionellen Formen künftiger Politik aufgelöst. Diese werden mit Demokratie, Technokratie und Autokratie bestimmt, welche eine je andere Art repräsentieren, mit der Ungewissheit der Zukunft umzugehen (wobei der Demokratiebegriff hier erneut sehr minimalistisch als ein akzeptiertes mehrheitliches, rekursives Wahlverfahren formuliert wird). Normativ und mit Blick auf die Wahrscheinlichkeit ihrer Durchsetzung werden die drei Formen dabei gleich behandelt (Baecker 2018, S. 105).

Von diesen systemtheoretischen Verständnissen setzen sich die kultursoziologischen Diagnosen klar ab, ohne aber institutioneller Politik ein größeres Eigengewicht zuzugestehen. Bei Stalder lässt sich eine De-Institutionalisierung des Politikbegriffs beobachten, indem er kollektiv gestaltende Handlungsform in kulturellen Praktiken aufgehen lässt. Zwar definiert er Demokratie institutionell als ,formalisierte, verbindliche und durch die Beteiligung der Bürger*innen legitimierte Entscheidungsfindung, die das Gemeinwesen betreffen“ (Stalder 2016, S. 209). Diese formalpolitisch Dimension spielt jedoch im Gang seines Arguments allenfalls in ihrer Abwesenheit eine Rolle. Sie ist ein Indikator des politischen Verfalls: Die Ausgestaltung der digitalen Medien ist weder bedingt durch einen technischen Imperativ noch Konsequenz politischen Handelns, sondern die Folge einer ,,politisch-ökonomisch-technischen Konstellation, welche die Möglichkeiten der Gegenwart (Produktivkräfte) in konkreten institutionellen Formen (Produktionsverhältnisse) realisiert, vorangetrieben im Interesse der Profit- und Kontrollmaximierung" (Stalder 2016, S. 209f.). Daraus folgt zweierlei: Stalder entpolitisiert die Kultur der Digitalität, da insbesondere ökonomisch-kulturelle Prozesse für die Ausgestaltung gesellschaftlicher Verhältnisse als relevant gesehen werden und er unterminiert die demokratische Normativität, wenn er zwar Demokratie als Norm ausruft, es faktisch aber mit einem libertären Thrasymachos hält: der Leistungfähigere setzt sich im unformalisierten rough consensus der Internetmeritokratie durch (Stalder 2016, S. 250).

Auch Reckwitz' Politikbegriff ist kulturalistisch geprägt: Bei ihm kommt Politik vor allem als Objekt kultureller Praktiken - und damit auch der Digitalisierung in den Blick. Die Auflösung der Politik des Allgemeinen in der ,Politik des Besonderen“ wird dabei als Ergebnis einer „Kulturalisierung der Politik“ beschrieben (Reckwitz 2017, S. 371). Diese resultiert in Formen der Identitätspolitik und des apertistisch-differenziellen Liberalismus, welche derivativ und häufig dem Genetiv verhaftet bleiben - die Politik der Städte, die Politik des Wettbewerbsstaates, die Politik der kulturellen Diversität usw. Ihre Dynamik gewinnt die gesellschaftliche Formierung dagegen durch die Ökonomie der Singularitäten, die „Kulturmaschine 
der digitalen Technologien“" und den singularistischen Lebensstil (Reckwitz 2017, S. 442). Indem Reckwitz, ähnlich wie Baecker und Nassehi, auch für die Zukunft der Politik die Fähigkeit zur Gestaltung abspricht und ihr ,höchstens indirekt[en]“ Einfluss zuschreibt (ebd.), bleibt schleierhaft, durch welche Verfahren und Institutionen seine retrotope Forderung nach einer Rückkehr zu Praktiken und Formen des Allgemeinen, eines doing universality, als Reaktion auf die „Krise des gemeinsam Geteilten“ (Reckwitz 2017, S. 440) eingelöst werden soll. Auch der Maßstab und die Quelle der Legitimität, anhand derer sich sein Vorschlag eines ,regulativen Liberalismus“ und dessen Umsetzung bewerten lassen müsste, bleibt damit unklar (Reckwitz 2017, S. 441).

\section{Politische Theorie der digitalen Konstellation}

Die einseitige Fokussierung der politikwissenschaftlichen Digitalisierungsforschung auf das Internet und Vernetzung und die deutlich umfassenderen, aber ein derivatives Verständnis des Politischen aufweisende Aufarbeitung der digitalen Gesellschaft in den Nachbardisziplinen, werfen die Frage auf, wie eine Politische Theorie der Digitalisierung denn anders gestaltet werden könnte. Diese müsste in der Lage sein, gesellschaftliche Wandlungsdynamiken umfassend und systematisch zu erfassen, andererseits aber die Spezifika demokratischer Politik und deren Gestaltungsmöglichkeiten berücksichtigen: Denn indem ,nicht sein muß, was ist“ (Blumenberg 1987, S. 57; vgl. Greven 2010), erhebt ein modernes Denken des Politischen den Anspruch auf eine zumindest relative Gestaltung sozialer Verhältnisse. In demokratischen Gesellschaften wird dies durch die Besonderheit komplementiert, dass nicht allein die technischen Möglichkeiten, sondern auch spezifische normative Anforderungen, etwa gleicher Berücksichtigung und freiheitlicher Selbstbestimmung, den Prozess sozialer Gestaltung strukturieren. Die Annahme einer relativen Gestaltungsfähigkeit kollektiver Handlungsformen bildet dabei aber die Voraussetzung politischer Reflexion und erst unter dieser Bedingung kann eine politikwissenschaftlich reflektierte Betrachtung der Digitalisierung - etwa hinsichtlich demokratischer Zentralkategorien wie Herrschaft, Partizipation, Repräsentation oder Öffentlichkeit - erfolgreich formuliert werden.

\subsection{Die digitale Konstellation}

Ziel des folgenden Vorschlags ist es daher, eine Beschreibung der Politik in der digitalen Konstellation zu liefern. Das heißt, die Bedingungen zu reflektieren, unter denen sich Politik in einer Gesellschaft vollzieht, die durch den Umgang mit digitaler Technik geprägt ist. Dies ist weniger als eine umfassende Theorie der digitalen Gesellschaft, aber doch mehr als die Internetforschung, welche für die Politikwissenschaft bisher charakteristisch ist. Digitalisierung würde dann nicht als Bereichsphänomen interpretiert, sondern als ein umfassender, vielgestaltiger Prozess, in dem sich Gesellschaft und Technik in einer konkreten Weise dynamisch und fortlaufend in Beziehung setzen. Versuche, beide Bereiche zu separieren oder auf ein einseitiges Wirkungsverhältnis im Sinne von , die Folgen der ... für ...“ zu reduzieren, 
laufen ins Leere. Von den rekonstruierten paradigmatischen Deutungsangeboten der Nachbardisziplinen lassen sich vielmehr zwei Dinge lernen: Zum einen besteht das Erfordernis, gesellschaftstheoretische Grundannahmen grundlegend zu reflektieren, da deren Transformation Einfluss nimmt auf Möglichkeiten und Praxis politischen Handelns in gesellschaftlicher wie institutioneller Form. Zum anderen braucht es ein Verständnis dessen, wie digitale Technik als Teil des kollektiven Vergesellschaftungsprozesses Bedingungen setzt, aber selbst auch geformt und bedingt wird, wie sich also entlang technischer Affordanzen Möglichkeitsräume eröffnen und soziales wie politisches Handeln realisiert wird.

Was es in der Perspektive der digitalen Konstellation zu untersuchen gilt, ist, wie sich in dieser Politik vollzieht. Also: Welche technischen Faktoren haben in welcher Weise eine prägende Bedeutung, welche Möglichkeitsräume oder Strukturlogiken bilden sich aus und verfestigen, wie Politik wiederum zurückwirkt auf Techniken, deren Entwicklung und gesellschaftliche Realisierung und schließlich, welche gesellschaftlichen oder politischen Praktiken setzen sich angesichts dieser sozio-technischen Umgebung durch und werden selbstverständlich? Der durch den Begriff der Konstellation bewusst gesetzte Bezug auf Jürgen Habermas (1998) und dessen Ende der 1990er Jahre geschriebene Auseinandersetzung mit der postnationalen Konstellation soll dabei unterstreichen, dass sich die Rahmenbedingungen politischen Handelns demokratischer Gesellschaften geändert haben, wodurch demokratische Politik anders zu reflektieren ist (vgl. auch: Pernice 2018; Hofmann 2019).

Angelehnt ist der Begriff hier an das philosophische Verfahren der Darstellung und des begrifflichen Erkenntnisgewinns, wie er als Konstellation oder Konfiguration etwa das Werk Theodor W. Adornos durchzieht. Dieser argumentiert, „daß man nicht einen argumentativen Zusammenhang in der üblichen Stufenfolge aufbauen kann, sondern daß man das Ganze aus einer Reihe von Teilkomplexen montieren muß [...]; deren Konstellation, nicht die Folge, muß die Idee ergeben." (Adorno 1970, S. 542; vgl. Lehr 2000). Dieses Vorgehen ist also gekennzeichnet durch ein diskursives Umkreisen, in welchem die Konstellation eines Begriffs in Relation zu anderen Begriffen zur Erfassung des Gegenstandes beitragen soll. Habermas überführt diese philosophische Logik in die Analyse einer sich verändernden historischen Konstellation, die ursprünglich zur institutionellen Ausprägung der Demokratie (bzw. der bürgerlichen Öffentlichkeit) geführt habe, und die nun in ihrem Wandlungsprozess die Bedingungen sowie die Normativität ihrer Formierung zu unterminieren drohe (Habermas 1998, S. 94; vgl. Habermas 1990, S. 148). In diesem Sinne ist auch die überwiegende politikwissenschaftliche Rezeption zu verstehen, wie sie hinsichtlich der Globalisierung etwa für die Fragen von Krieg und Frieden (Zangl und Zürn 2003, S. $149 \mathrm{ff}$.) oder Souveränität (Volk und Kuntz 2014) thematisiert wurde. Gemein ist diesen, wie auch unserem eigenen Zugriff, die Annahme, dass eine veränderte gesellschaftliche Konstellation bisher geltende Faktoren in eine neue Relation setze, womit die Notwendigkeit einer Anpassung analytischer wie normativer Denkmuster einhergeht.

Um den Untersuchungsgegenstand - in unserem Fall also die digitale Konstellation - analytisch zu differenzieren, schlagen wir vor, zunächst drei Ebenen zu unterscheiden, auf denen Digitalisierung relevant wird: die Ebene der Eigenschaf- 
ten digitaler Technik, die Ebene praktisch realisierter Affordanzen und die Ebene der gesellschaftlichen Konfiguration bzw. des politischen Formwandels. Die ,Eigenschaften' digitaler Technik sind wie schon bei den soziologischen Autoren über Datafizierung - das Umwandeln analoger in digitale Information - zu rekonstruieren, wobei besonders hervorstechend die Archivierbarkeit, Vernetzbarkeit und Prozessierbarkeit (Algorithmisierung) digitaler Daten zu nennen sind (vgl. ähnlich Stalder 2016; Lenk 2016). Diese abstrakten Eigenschaften bilden eine Konstante, die in Verbindung mit Handlungspraktiken und -kontexten unterschiedliche Ausprägungen annehmen kann. Eine zweite Ebene der Beschreibung ist die Reihe praktisch realisierter Affordanzen, also eine generalisierte Aussage über kollektiv etablierte Handlungsformen, die sich aus den wahrgenommenen Möglichkeitsräumen digitaler Technik ergeben. Hinter dem Konzept der Affordanzen steht die Überlegung, dass Technik soziale Strukturen nicht determiniert, sondern Angebotsstrukturen für Nutzungspraktiken bereitstellt, sprich: Handlungsmöglichkeiten eröffnet (Hutchby 2001, S. 444; Latour 2017, S. 124). Affordanzen werden von uns im Anschluss an Evans et al. konzeptuell als facettenreiche Beziehungsstruktur zwischen einem technischen Artefakt und dessen Nutzer*in verstanden, welche in einer konkreten Situation potentielle Handlungsergebnisse ermöglicht oder beschränkt (Evans et al. 2017, S. 36). Die Perspektive richtet den Analysefokus auf den Zusammenhang von Technik und sozialen Praktiken, ist dabei aber auch mit Grundüberlegungen politischen Denkens - etwa Freiheitlichkeit und Kontingenz politischen Handelns vereinbar. Während sich Affordanzen einer Technik immer auf konkrete Situationen beziehen und abhängig sind von dem dort existierenden, subjektiv wahrgenommenen Kontext, geht es uns in der Beschreibung praktisch realisierter Affordanzen aber politikwissenschaftlich geweitet um eine Generalisierung derjenigen Affordanzen, die unter ähnlichen Bedingungen in ähnlicher Weise verwirklicht wurden und aus denen sich reflektierbare, gesamtgesellschaftliche Pfadabhängigkeiten ergeben. Ein Beispiel für eine solche praktisch realisierte Affordanz digitaler Technik ist die $M a$ ny-to-Many-Kommunikation. Affordanzen können so verstanden als theoretisches Element in nicht-deterministischer Weise Aussagen über den Zusammenhang von digitaler Technik und sozialer oder politischer Formation ermöglichen. Auf der dritten Ebene schließlich lassen sich Aussagen über die gesellschaftliche Konfiguration als Resultat soziotechnischer Wandlungsprozesse tätigen. Technische Eigenschaften und politische Dynamiken sind dann in der Adaption fest miteinander verbunden und bringen relativ stabile gesellschaftlich-strukturelle Phänomene hervor wie etwa die gegenwärtig realisierte Plattformökonomie.

Damit der Wert des Vorgehens über die digitale Konstellation sowie der skizzierten Unterscheidung der drei Ebenen deutlich wird, wenden wir uns nun abschließend noch einmal illustrativ einem besonders hervorgehobenen Bereich der demokratischen Politik zu, der politischen Repräsentation, und zeigen, wie man unsere Perspektive analytisch produktiv und politikwissenschaftlich relevant zum Einsatz bringt. 


\subsection{Politische Repräsentationsdynamiken zwischen konnektiver Organisation und datenbasierter Analyse}

Der Formwandel respektive die Krise demokratischer Repräsentation ist kein neues Phänomen, vielmehr stellt die „Quadratur des Zirkels“ eine demokratiegeschichtliche Konstante dar, die sich in der Angleichung normativer Ideale wie Freiheit und Gleichheit, institutioneller Formen und gesellschaftlicher Praktiken immer wieder aufs Neue stabilisiert (Landshut 1968, S. 490). Dennoch lassen sich unschwer digitalisierungsbezogene Entwicklungen ausmachen, die auf eine strukturelle Überformung des Verständnisses, der Praxis und der Institution politischer Repräsentation, des ,democratic interface“, hindeuten (Bennett et al. 2018; Karlsson 2013).

Augenfällig ist dies im Bedeutungsgewinn konnektiver Formen der Parteiorganisation oder im Aufkommen neuer digitaler Parteien und Bewegungen von den Piraten über das MoVimento 5 Stelle hin zu Tea Party oder Brand New Congress. Digitale Techniken ermöglichen niedrigschwellige Vernetzung sowie eine affektiv aufgeladene und sehr unmittelbare Kommunikation innerhalb und über die Organisation hinaus. In ihrem Kontext verändern sich Beteiligungserwartungen und -präferenzen. Gerade traditionelle Formen kollektiver Organisation mittels hierarchisch organisierter Strukturen geraten so gegenüber deutlich durchlässigeren, intern heterogenen und an die technische Formation plattformbasierter Kommunikation erinnernde Parteien und Bewegungen ins Hintertreffen (Gerbaudo 2019) - obschon bezweifelt werden kann und sollte, dass aus diesen horizontal-partizipativen Elementen bereits per se Egalisierung oder Demokratisierung folgt (Urbinati 2015; Kelty 2015).

Die politikwissenschaftliche Analyse darf hier nun aber nicht enden; sie muss vielmehr weitere Effekte der digitalen Konstellation auf politische Repräsentation aufdecken und in die Analyse einbeziehen. So erlaubt eine digitalisierte Kommunikationsumgebung nicht nur direkteren Austausch, das Interaktionssverhältnis kehrt sich vielmehr auch von der anderen Seite her um. Hierfür steht der als analytics turn bezeichnete Trend, durch die automatisierte Analyse großer Datenmengen Informationen über Verhalten und Präferenzen der Bürger*innen zu sammeln, Profile zu generieren und dann wiederum das politische Handeln der Repräsentant*innen darauf auszurichten (Chadwick und Stromer-Galley 2016, S. 284). Der Logik nach zwar schon älter - nämlich auf die Quantifizierung der öffentlichen Meinung und die Meinungsforschung zurückgehend (Herbst 1993; Keller 2007) - eröffnet sich unter den Bedingungen der digitalen Konstellation die dann auch wahrgenommene Möglichkeit, in exponentiell vergrößertem Maßstab und mittels algorithmischer Analyse öffentliche Meinung umfassend zu rekonstruieren, zugleich aber auch Politik weiter zu individualisieren (McGregor 2019; Tufekci 2014). Im Angesicht komplexer und unsicherer Repräsentationsverhältnisse reagieren „mathematisch geschulte Menschen entsprechend mit dem ,rationalen Reflex ', nach mehr Daten und noch komplexeren Analysemodellen zu verlangen um ja keinen möglicherweise relevanten Aspekt zu übersehen“ (Neth und Gaissmaier 2017, S. 214). Hier wird das Zusammenspiel von Eigenschaften digitaler Technik - Archivierbarkeit, Prozessierbarkeit - mit der darauf begründeten Möglichkeit - automatisierte Mustererkennung, Profilbildung, Personalisierung - und auch wieder der Wirkung von Politik auf die Technikentwicklung deutlich. Das daraus resultierende ,,passive democratic feedback“ (Karpf 
2012, S. 23, 2016) etabliert sich als Element politischer Willensbildung, das demokratietheoretisch noch überhaupt nicht eingeholt ist, während zugleich soziale Netzwerke oder Apps Kampagnen und Repräsentant*innen den ,direkten“ Kontakt mit Bürger*innen ermöglichen. Formen des „,computational management“" (Kreiss 2012, S. 24) und der „controlled interactivity“ (Stromer-Galley 2014, S. 104ff.) der Repräsentationsbeziehung bilden einen Kontrapunkt zur partizipativ-emanzipativen Lesart des digitalen Wandels politischer Repräsentation - ohne einfach nur deren Widerlegung darzustellen.

Wir beobachten an dieser Stelle also, wie eine Adaption der durch die technischen Möglichkeitsräume digitaler Medien gezeichneten digitalen Konstellation sich vollzieht, aber auch, wie der Hintergrund der Repräsentationskrise als Legitimation und Katalysator für das Experiment mit neuen Politikformen dient (König 2018; Chadwick und Stromer-Galley 2016; früh bereits: Margetts 2001; Gellner und Strohmeier 2002). ${ }^{7}$ Dass Adaptionsprozesse uneinheitlich und wiederum geprägt von Faktoren wie der politischen Kultur, dem Handeln politischer Eliten und den rechtlichen und strukturellen Rahmenbedingungen sind, zeigt sich deutlich, wenn man Themen wie Microtargeting dann wieder in unterschiedlichen politischen Bezugsräumen wie den USA und Deutschland vergleicht (Bennett 2016; Jungherr 2016; vgl. Zuiderveen Borgesius et al. 2018; Kruschinski und Haller 2017).

Unterschiede und Wechselwirkungen differenziert aufzuarbeiten heißt nicht, auf übergreifende Aussagen über die digitale Konstellation verzichten zu müssen. Diese lassen sich trotzdem rekonstruieren, müssen nun aber die stabilisierenden wie dynamisierenden Momente explizit reflektieren. Dazu zählt etwa die kritische Einsicht, dass digitale Interaktivität Affordanzen herausgebildet hat, die einen populistischen Modus der Politik verstärken und die auf der Ebene der gesellschaftlichen Konfiguration stabilisiert werden - etwa durch die werbetechnisch geprägte Plattformökonomie als Infrastruktur politischer Interaktion. Die demokratische Fokussierung auf das Volk und den Volkswillen führt unter den Bedingungen der digitalen Konstellation - ihren Anreizen, dass die Bürger*innen permanent und differenziert ausgelesen, befragt, und wiederum überzeugt werden können - sowohl in der skizzierten konnektiven wie analytischen Rekonstruktion zu einer spezifischen Entwicklung von Repräsentation. In dieser dominiert eine instrumentelle, aber nicht passivierende Perspektive auf die individuellen Wähler*innen und ein Politikstil, der den Kampf um Authentizität und Rechenschaftspflicht durch eine möglichst kongruente Übernahme eines sich unmittelbar digital manifestierenden Volkswillen prämiert - freilich ohne, dass daraus zwingend eine Einlösung demokratischer Responsivitätsversprechen resultieren würde (Baldwin-Philippi 2018). Hinzu kommt, dass ein solches Verständnis responsiver Repräsentation durch plebiszitäre und identitätstheoretische Konzepte und Verfahren geprägt ist, welches nicht nur die handlungs- und differenztheoretischen Grundlagen parlamentarischer Repräsentation unterminiert (Thaa

\footnotetext{
7 Auch repräsentationstheoretisch sind die Bedürfnisse einer informationstechnischen Anbindung von Wähler*innen bzw. Bürger*innen und Repräsentant*innen bereits in den 80er Jahren diskutiert worden, damals noch vor dem Hintergrund einer modernisierungstheoretischen Steuerungs- und Komplexitätskrise. Die Vorschläge „kybernetischer Repräsentation“ lassen sich daher als konzeptuelle Vorläufer in der Verbindung repräsentativer Institutionen und informationstechnischer Strukturen verstehen (Herzog 1989; Mirbach 1992).
} 
2016), sondern auch die Funktionslogik - und damit die Legitimität - repräsentativer Institutionen zur Disposition stellt (Urbinati 2019). In einer solchen Erklärung ist es dann nicht die durch Digitalisierung induzierte Komplexität der Politik, welche die Demokratie heraus- bzw. überfordert. Stattdessen muss die Entwicklung der Demokratie vielmehr zumindest auch aus der Entfaltung politischer Praxis gedeutet werden. Politikwissenschaft im Allgemeinen und Politische Theorie im Besonderen sind daher gut beraten, die technische Dimension in ihrem Wechselspiel mit politischen Formierungen zu reflektieren, wollen sie begründete Aussagen treffen und nicht die Wirkungszusammenhänge als ,Black Box‘ beschreiben.

\section{Abschließende Bemerkungen}

Dieses Beispiel einer exemplarischen Analyse der demokratischen Entwicklung im Zeichen der digitalen Konstellation zeigt bereits, dass Politikwissenschaft mehr zum Diskurs beitragen kann als sie es derzeit tut. Es zeigt aber auch, dass die disziplinären Fragestellungen sich wandeln, wenn die technischen Bedingungen explizit in die Auseinandersetzung einbezogen werden. Nötig ist es, ein Verständnis von Digitalisierung und digitaler Gesellschaft zu entwickeln, welches die Wirkweise von Technik und Medien in Gesellschaft auch für politische Zusammenhänge erschließt. Unser Vorschlag dafür ist ein doppelter: Zum einen Digitalisierung als digitale Konstellation zu fassen und so auf die Möglichkeiten und den Prozesscharakter abzustellen; zum anderen die Wirkweise von Technik - hier konkret Digitalisierung mehrschichtig und nicht-deterministisch zu konzeptualisieren. Politikwissenschaft und Politische Theorie bzw. Demokratietheorie müssen hierfür nicht auf die Detailebene einzelner Techniken einsteigen, sondern können durchaus generalisierend Zusammenhänge erforschen. Dabei gilt es jedoch, ein komplexes Verständnis der Wechselwirkungen von Technik und Gesellschaft zu gewinnen, ohne den Blick für die Spezifika der politischen Organisation von Gesellschaft und besonders deren demokratischen Institutionen zu verlieren. Hierbei kann man aus dem Dialog mit den Nachbardisziplinen viel lernen - und diesen selbst auch bereichern.

Funding Open Access funding provided by Projekt DEAL.

Open Access Dieser Artikel wird unter der Creative Commons Namensnennung 4.0 International Lizenz veröffentlicht, welche die Nutzung, Vervielfältigung, Bearbeitung, Verbreitung und Wiedergabe in jeglichem Medium und Format erlaubt, sofern Sie den/die ursprünglichen Autor(en) und die Quelle ordnungsgemäß nennen, einen Link zur Creative Commons Lizenz beifügen und angeben, ob Änderungen vorgenommen wurden.

Die in diesem Artikel enthaltenen Bilder und sonstiges Drittmaterial unterliegen ebenfalls der genannten Creative Commons Lizenz, sofern sich aus der Abbildungslegende nichts anderes ergibt. Sofern das betreffende Material nicht unter der genannten Creative Commons Lizenz steht und die betreffende Handlung nicht nach gesetzlichen Vorschriften erlaubt ist, ist für die oben aufgeführten Weiterverwendungen des Materials die Einwilligung des jeweiligen Rechteinhabers einzuholen.

Weitere Details zur Lizenz entnehmen Sie bitte der Lizenzinformation auf http://creativecommons.org/ licenses/by/4.0/deed.de. 


\section{Literatur}

Adorno, Theodor W. 1970. Gesammelte Schriften 7. Ästhetische Theorie. Frankfurt am Main: Suhrkamp.

August, Vincent. 2018an. Technologisches Regieren: Der Aufstieg des Netzwerk-Denkens in der Krise der Moderne Foucault, Luhmann und die Kybernetik. Dissertation. Berlin: Humboldt-Universität zu Berlin.

Baecker, Dirk. 2018. 4.0 oder Die Lücke die der Rechner lässt. Leipzig: Merve Verlag.

Baldwin-Philippi, Jessica. 2018. The technological performance of populism. New Media \& Society 21(2):376-397

Barber, Benjamin R. 1998. Three scenarios for the future of technology and strong democracy. Political Science Quarterly 113(4):573-589.

Bennett, Colin J. 2016. Voter databases, micro-targeting, and data protection law: can political parties campaign in europe as they do in north America? International Data Privacy Law 6(4):261-275.

Bennett, W. Lance, und Alexandra Segerberg. 2014. The logic of connective action: digital media and the personalization of contentious politics. Cambridge: Cambridge University Press.

Bennett, W. Lance, Alexandra Segerberg, und Curd Knüpfer. 2018. The democratic interface: technology, political organization, and diverging patterns of electoral representation. Information, Communication \& Society 21(11):1655-1680.

Bieber, Christoph, und Claus Leggewie (Hrsg.). 2012. Unter Piraten: Erkundungen in einer neuen politischen Arena. Bielefeld: transcript.

Blumenberg, Hans. 1987. Die Sorge geht über den Fluß. Frankfurt am Main: Suhrkamp.

Bohman, James. 2004. Expanding dialogue: the internet, the public sphere and prospects for transnational democracy. The Sociological Review 52:131-155.

Buchstein, Hubertus. 1996. Bittere Bytes: Cyberbürger und Demokratietheorie. Deutsche Zeitschrift für Philosophie 44(4):583-608.

Busch, Andreas, Yana Breindl, und Tobias Jakobi (Hrsg.). 2019. Netzpolitik: Ein einführender Überblick. Wiesbaden: VS.

Celikates, Robin. 2015. Digital publics, digital contestation: a new structural transformation of the public sphere? In Transformations of democracy: crisis, protest and legitimation, Hrsg. Timo Wesche, Regina Kreide, 159-176. London: Rowman \& Littlefield.

Chadwick, Andrew, und Jennifer Stromer-Galley. 2016. Digital media, power, and democracy in parties and election campaigns: party decline or party renewal? The International Journal of Press/Politics 21(3):283-293.

Couldry, Nick, und Andreas Hepp. 2016. The mediated construction of reality. Cambridge, Malden: Polity.

Dahlberg, Lincoln. 2011. Re-constructing digital democracy: an outline of four 'positions'. New Media \& Society 13(6):855-872.

Dahlgren, Peter. 2009. Media and political engagement: citizens, communication and democracy. Cambridge: Cambridge University Press.

Dean, Jodi. 2003. Why the net is not a public sphere. Constellations 10(1):95-112.

Deutsch, Karl Wolfgang. 1969. Politische Kybernetik, Modelle und Perspektiven. Freiburg: Rombach.

Earl, Jennifer, und Katrina Kimport. 2011. Digitally enabled social change: activism in the internet age. Cambridge: MIT Press.

Eifert, Martin, und Wolfgang Hoffmann-Riem (Hrsg.). 2009. Innovation und Recht II: Innovationsfördernde Regulierung. Berlin: Duncker \& Humblot.

Evans, Sandra K., Katy E. Pearce, Katy E.Jessica Vitak, und Jeffrey W. Treem. 2017. Explicating affordances: a conceptual framework for understanding affordances in communication research. Journal of Computer-Mediated Communication 22(1):35-52.

Floridi, Luciano. 2015. Die 4. Revolution: Wie die Infosphäre unser Leben verändert. Berlin: Suhrkamp.

Fries, Martin. 2016. PayPal Law und Legal Tech - Was macht die Digitalisierung mit dem Privatrecht? NJW 2016:2860-2865.

Gellner, Winand, und Gerd Strohmeier. 2002. Cyber-Kampagnen. In Wahl-Kämpfe: Betrachtungen über ein demokratisches Ritual, Hrsg. Andreas Dörner, Ludgera Vogt, 164-186. Frankfurt am Main: Suhrkamp.

Gerbaudo, Paolo. 2019. The digital party: political organisation and Online democracy. London: Pluto Press.

Greven, Michael T. 2010. Verschwindet das Politische in der politischen Gesellschaft? Über Strategien der Kontingenzverleugnung. In Das Politische der Politik, Hrsg. Thomas Bedorf, Kurt Röttgers, 68-101. Berlin: Suhrkamp. 
Habermas, Jürgen. 1990. Strukturwandel der Öffentlichkeit. Frankfurt am Main: Suhrkamp.

Habermas, Jürgen. 1998. Die postnationale Konstellation: Politische Essays. Frankfurt am Main: Suhrkamp.

Habermas, Jürgen. 2008. Hat die Demokratie noch eine epistemische Dimension? Empirische Forschung und normative Theorie. In Ach, Europa, Hrsg. Jürgen Habermas, 138-191. Frankfurt am Main: Suhrkamp.

Herbst, Susan. 1993. Numbered Voices. How Opinion Polling Has Shaped American Politics. Chicago, London: The University of Chicago Press.

Herzog, Dietrich. 1989. Was heißt und zu welchem Ende studiert man Repräsentation? In Konfliktpotentiale und Konsensstrategien. Beitrage zur politischen Soziologie der Bundesrepublik, Hrsg. Dietrich Herzog, Bernhard Weßels, 307-335. Opladen: Westdeutscher Verlag.

Hindman, Matthew. 2008. The myth of digital democracy. Princeton: Princeton University Press.

Hofmann, Jeanette. 2019. Mediated democracy - linking digital technology to political agency. Internet Policy Review 8(2). https://doi.org/10.1177/1461444818797591.

Howard, Philip N. 2010. The digital origins of dictatorship and democracy. Oxford, New York: Oxford University Press.

Hutchby, Ian. 2001. Technologies, texts and affordances. Sociology 35(2):441-456.

Ingold, Albert. 2017. Digitalisierung demokratischer Öffentlichkeiten. Der Staat 56(4):491-533.

Jäckel, Michael, und Uwe Jun (Hrsg.). 2015. Wandel und Kontinuität der politischen Kommunikation. Opladen: Barbara Budrich.

Jacob, Daniel, und Thorsten Thiel (Hrsg.). 2017. Politische Theorie und Digitalisierung. Baden-Baden: Nomos.

Jungherr, Andreas. 2016. Four functions of digital tools in election campaigns: the German case. The International Journal of Press/Politics 21(3):358-377.

Jungherr, Andreas, und Yannis Theocharis. 2017. The empiricist's challenge: asking meaningful questions in political science in the age of big data. Journal of Information Technology \& Politics 14(2):97-109.

Karlsson, Martin. 2013. Representation as interactive communication: theoretical considerations and empirical findings. Information, Communication \& Society 16(8):1201-1222.

Karpf, David. 2016. Analytic activism: digital listening and the new political strategy. New York: Oxford University Press.

Karpf, David. 2012. Will the real psychometric targeters please stand up? Civic hall. https:/civichall.org/ civicist/will-the-real-psychometric-targeters-please-stand-up/. Zugegriffen: 24. Mai 2019.

Keane, John. 2013. Democracy and media decadence. Cambridge: Cambridge University Press.

Keller, Felix. 2007. Figuren des Publikums: Politischer und diagrammatischer Raum. In Formation der Mediennutzung II, Hrsg. Irmela Schneider, 153-170. Bielefeld: transcript.

Kelty, Christopher, Aaron Panofsky, Morgan Currie, Roderic Crooks, Seth Erickson, Patricia Garcia, Michael Wartenbe, und Stacy Wood. 2015. Seven Dimensions of Contemporary Participation Disentangled. Journal of the Association for Information Science and Technology 66(3):474-488.

Kersten, Jens. 2017. Schwarmdemokratie. Der digitale Wandel des liberalen Verfassungsstaats. Tübingen: Mohr Siebeck.

Kersting, Norbert. 2019. Digitalization and political science in Germany. In Political science and digitalization-global perspectives, Hrsg. Helen V. Milner, Marianne Kneuer. Opladen: Barbara Budrich.

Kleger, Heinz, und Eric Makswitat. 2014. Digitaler Ungehorsam Wie das Netz den zivilen Ungehorsam verändert. Forschungsjournal Soziale Bewegungen 27(4):8-17.

Kneuer, Marianne (Hrsg.). 2013. Das Internet: Bereicherung oder Stressfaktor für die Demokratie? BadenBaden: Nomos.

Kneuer, Marianne, und Samuel Salzborn (Hrsg.). 2016. Web 2.0 - Demokratie 3.0: Digitale Medien und ihre Wirkung auf demokratische Prozesse. Wiesbaden: VS.

König, Pascal D. 2018. Algorithmen und die Verwaltung sozialer Komplexität: Zur Neukonfigurierung der Idee der Selbstregierung des Volkes. Zeitschrift für Politikwissenschaft 28(3):289-312.

Kreiss, Daniel. 2012. Taking our country back: the crafting of networked politics from Howard Dean to Barack Obama. New York: Oxford University Press.

Kruschinski, Simon, und André Haller. 2017. Restrictions on data-driven political micro-targeting in Germany. Internet Policy Review 6(4). https://doi.org/10.14763/2017.4.780.

Landshut, Siegfried. 1968. Der politische Begriff der Repräsentation. In Zur Theorie und Geschichte der Repräsentation und Repräsentativverfassung, Hrsg. Heinz Rausch, 482-497. Darmstadt: WBG.

Latour, Bruno. 2017. Eine nеuе Soziologie für eine nеue Gesellschaft. Berlin: Suhrkamp. 
Lehr, Andreas. 2000. Kleine Formen. Adornos Kombinationen: Konstellation/Konfiguration, Montage und Essay. Dissertation. Freiburg i. Br.: Albert-Ludwigs-Universität.

Lenk, Klaus. 2016. Die neuen Instrumente der weltweiten digitalen Governance. Verwaltung \& Management 22(5):227-240.

Margetts, Helen. 2001. The Cyber party. Paper to workshop 'the causes and consequences of Organisational innovation in European political parties. ECPR Joint Sessions of Workshops, Grenoble, 6-11 April 2001.

Margetts, Helen. 2013. The internet and democracy. In The oxford handbook of Internet studies, Hrsg. William H. Dutton. Oxford: Oxford University Press.

Margetts, Helen. 2019. Rethinking democracy with social media. The Political Quarterly 90(S1):107-123.

McGregor, Shannon C. 2019. Social media as public opinion: How journalists use social media to represent public opinion. Journalism 20(8):1070-1086.

Mirbach, Thomas. 1992. Eine kybernetische Auflösung des Repräsentationsproblems? Zeitschrift für Parlamentsfragen 23(4):658-672.

Nassehi, Armin. 2019. Muster: Eine Theorie der digitalen Gesellschaft. München: C.H. Beck.

Neth, Hansjörg, und Wolfgang Gaissmaier. 2017. Warum erfolgreiche Prognosen einfach und unsicher sind: Von der Wahl des richtigen Werkzeugs für Wähler und die Wahlforschung. Zeitschrift für Politikwissenschaft 27(2):205-220.

Pernice, Ingolf. 2018. Stärkung der Demokratie in der Digitalen Konstellation. In Festschrift für Matthias Schmidt-Preuss zum 70. Geburtstag, Hrsg. Markus Ludwigs. Berlin: Duncker \& Humblot.

Pohle, Julia, und Thorsten Thiel. 2019. Digitale Vernetzung und Souveränität: Genealogie eines Spannungsverhältnisses. In Internet und Staat, Hrsg. Isabelle Borucki, Wolf Schünemann, 57-78. BadenBaden: Nomos.

Reckwitz, Andreas. 2017. Die Gesellschaft der Singularitäten: Zum Strukturwandel der Moderne. Berlin: Suhrkamp.

Reiberg, Abel. 2018. Netzpolitik. Genese eines Politikfeldes. Baden-Baden: Nomos.

Ritzi, Claudia, und Alexandra Zierold. 2019. Souveränität unter den Bedingungen der Digitalisierung. In Internet und Staat, Hrsg. Isabelle Borucki, Wolf Schünemann. Baden-Baden: Nomos.

Schmalz-Bruns, Rainer. 2001. Internet-Politik. Zum demokratischen Potenzial der neuen Informationsund Kommunikationstechnologien. In Politik und Technik Politische Vierteljahresschrift 31., Hrsg. G. Simonis, R. Martinsen, und T.T. Saretzki. Wiesbaden: VS.

Schünemann, Wolf, und Marianne Kneuer. 2019. E-Government und Netzpolitik im europäischen Vergleich. Baden-Baden: Nomos.

Seubert, Sandra. 2016. Emanzipation und Beherrschung. Zur Kritik des Privaten im digitalen Zeitalter. WestEnd 14(1):89-103.

Stahl, Titus. 2016. Indiscriminate mass surveillance and the public sphere. Ethics and Information Technology 18(1):33-39.

Stalder, Felix. 2016. Kultur der Digitalität. Berlin: Suhrkamp.

Steiger, Stefan, Wolf Schünemann, und Katharina Dimmroth. 2017. Outrage without consequences? PostSnowden discourses and governmental practice in Germany. Media and Communication 5(1):7-16.

Stromer-Galley, Jennifer. 2014. Presidential Campaigning in the Internet Age. Oxford: Oxford University Press.

Sunstein, Cass R. 2008. Democracy and the Internet. In Information technology and moral philosophy, Hrsg. J. Van den Hoven, J. Weckert, 93-110. Cambridge: Cambridge University Press.

Thaa, Winfried. 2016. Die Repräsentation von Differenz als Voraussetzung politischen Handelns. In Die Aktualität des Republikanismus, Hrsg. Thorsten Thiel, Christian Volk, 73-93. Baden-Baden: Nomos.

Thiel, Thorsten. 2017. Anonymität und Demokratie. Forschungsjournal Soziale Bewegungen 30(2):152-161.

Tufekci, Zeynep. 2014. Engineering the public: big data, surveillance and computational politics. First Monday 19(7). https://doi.org/10.5210/fm.v19i7.4901.

Tufekci, Zeynep. 2017. Twitter and tear gas: the power and fragility of networked protest. New Haven: Yale University Press.

Ulbricht, Lena, et al. 2018. Dimensionen von Big Data: Eine politikwissenschaftliche Systematisierung. In Big Data und Gesellschaft: Eine multidisziplinäre Annäherung, Hrsg. Barbara Kolany-Raiser, Reinhard Heil, Carsten Orwat, und Thomas Hoeren, 151-231. Wiesbaden: Springer.

Urbinati, Nadia. 2015. A revolt against intermediary bodies. Constellations 22(4):477-486.

Urbinati, Nadia. 2019. Me, the People. Cambridge, London: Harvard University Press.

Vesting, Thomas. 2015. Die Medien des Rechts: Computernetzwerke. Weilerswist: Velbrück Wissenschaft. Volk, Christian, und Friederike Kuntz (Hrsg.). 2014. Der Begriff der Souveränität in der transnationalen Konstellation. Baden-Baden: Nomos. 
Zangl, Bernhard, und Michael Zürn. 2003. Frieden und Krieg. Sicherheit in der nationalen und postnationalen Konstellation. Frankfurt am Main: Suhrkamp.

Zuboff, Shoshana. 2018. Das Zeitalter des Überwachungskapitalismus. Frankfurt am Main: Campus.

Zuiderveen Borgesius, Frederik J., Judith Möller, Sanne Kruikemeier, Ronan Ó. Fathaigh, Kristina Irion, Tom Dobber, Balazs Bodo, und Claes De Vreese. 2018. Online political microtargeting: promises and threats for democracy. Utrecht Law Review 14(1):82-96. 